\title{
Étude du comportement, au cours de l'embryogenèse somatique, de divers porte-greffes de vigne cultivés in vitro en conditions chlorosantes
}

\author{
MH Netzer, V Guellec, M Branchard \\ Université Paris XI, bâtiment 360, laboratoire d'amélioration des plantes, 91405 Orsay cedex, France
}

(Reçu le 18 juillet 1990; accepté le 29 novembre 1990)

\begin{abstract}
Résumé - Pour l'étude du comportement de porte-greffes de vigne en condition de chlorose calcaire, des ions bicarbonate sont ajoutés à différentes concentrations aux milieux de culture et à divers stades du processus d'embryogenèse somatique. Les anthères sont plus sensibles à la présence de ces ions que les cals embryogènes et, plus la concentration en ions bicarbonate est élevée, moins le développement se réalise. Leur addition est la cause d'une alcalinisation du milieu à laquelle la vigne réagit, d'une part, en acidifiant progressivement le milieu de culture, d'autre part en formant des cellules épidermiques à paroi épaisse, dans la zone apicale des racines.

Les résultats obtenus lors du test expérimental permettent le classement de sensibilité des porte-greffes à la chlorose calcaire voisin de celui observé au champ : 41B < $309 \mathrm{C}<$ Rupestris du Lot.
\end{abstract}

embryogenèse somatique / ion bicarbonate / modification racinaire / acidification du milieu de culture

Summary - Behaviour of grapevine rootstocks during somatic embryogenesis, under conditions of calcareous chlorosis. Different concentrations of $\mathrm{KHCO}_{3}$ were added to the culture media and to different steps of somatic embryogenesis with the aim of studying behaviour of grapevine rootstocks under conditions of calcareous chlorosis. Anthers were found to be more sensitive to $\mathrm{KHCO}_{3}$ than embryogenic calli and high concentrations $\left(>500 \mathrm{mg} . \mathrm{r}^{-1}\right)$ induced anther necrosis (table l). The higher the concentration of bicarbonate, the greater was the inhibition of development. The addition of $\mathrm{KHCO}_{3}$ induced high $\mathrm{pH}$ values in the medium (table III) to which the grapevine reacted by progressive acidification of the medium (fig 3 ) and the formation of thick-walled epidermal cells in the root tips (fig $5 A, B$ ). According to the results obtained in this experiment, the classification of rootstock sensitivity to calcareous chlorosis is: $41 B<3309$ C $<$ Rupestris du Lot.

somatic embryogenesis / bicarbonate / root transformation / medium / acidification

\section{INTRODUCTION}

La chlorose calcaire (ou chlorose ferrique) est une maladie physiologique qui se manifeste dans les sols calcaires. Dans ces types de sol, la présence d'acide carbonique provenant de l'eau du sol et du gaz carbonique issu de la respiration des racines, conduit, en présence de carbonate de calcium, à la libération massive d'ions bicarbonate. Chez la vigne, dès 1969, Saglio puis Juste et Pouget (1972) et Galet (1976) se sont intéressés à ce problème viticole. Par ailleurs, dès 1971, Brown et al, puis, plusieurs auteurs (Boxma, 1972; Pouget et Ottenwaelter, 1973; Coulombe et al, 1984a; Mayer, 1985; Fairbanks et al, 1987; Murisier et Briguet, 1988) chez différentes espèces (soja, vigne), soulignent le rôle de ces ions, qu'ils jugent directement responsables de la chlorose calcaire. Dans les conditions de $\mathrm{pH}$ élevé du sol, ces ions entraînent le maintien du fer à l'état oxydé $\left(\mathrm{Fe}^{3+}\right)$. Le fer est alors difficilement absorbable par les racines et demeure insoluble dans la plante, ce qui provoque une carence en fer, principalement au niveau du système foliaire, responsable, en particulier, de la perturbation de la synthèse de la chlorophylle.

Dans le cadre de la recherche de conditions in vitro permettant de simuler les conditions chlorosantes observées au champ, Chiadmi (1986) a 
mis au point, avec différents génotypes, un dispositif expérimental basé sur l'utilisation de boutures de porte-greffes. Divers paramètres sont mesurés $(\mathrm{pH}$ du milieu, dénombrement foliaire, absorption du fer). La complexicité des mesures effectuées ne permet pas leur utilisation routinière dans le cadre d'un test précoce de sélection. En conséquence, un nouveau schéma, où la pression de sélection est appliquée au cours du processus d'embryogenèse somatique, a été développé.

\section{MATÉRIEL ET MÉTHODES}

Différents porte-greffes de vigne présentant, au champ, des sensibilités différentes à la chlorose calcaire, sont utilisés en vue de l'obtention d'embryons somatiques par culture d'anthères : 41B-Rupestris du Lot -101.14 et 3309 Couderc.

Les sarments sont stockés au froid $\left(4^{\circ} \mathrm{C}\right)$ puis forcés en serre, selon la méthode mise au point par Mullins (1966). Les boutons floraux prélevés sur les inflorescences sont stérilisés à l'hypochlorite de calcium à $7 \%$ pendant 20 min puis rincés 3 fois à l'eau distillée. Trente fleurs sont ensuite disséquées et les anthères (5-6 par fleur) sont mises en culture dans un Erlenmeyer contenant $25 \mathrm{ml}$ du milieu liquide de Nitsch et Nitsch (1969) renfermant 1 mg.l-1 de 2,4-D; 0,25 mg..$^{-1}$ de BAP et 1 g. $\left.\right|^{-1}$ d'hydrolysat de caséine. La culture est maintenue en agitation $(80 \mathrm{rp} \mathrm{m}$ ) à l'obscurité, à une température de $27^{\circ} \mathrm{C} \pm 1{ }^{\circ} \mathrm{C}$, durant 4 semaines. Pour toutes les cultures, le milieu est alors éliminé puis remplacé par un milieu de même composition, mais sans régulateur de croissance et renouvelé chaque semaine. Les conditions de température sont identiques à celles précédemment définies mais les cultures sont alors exposées à un éclairement de $50 \mu \mathrm{E} \cdot \mathrm{m}^{-2} . \mathrm{s}^{-1}$ (tubes Gro-Lux Sylvania) avec une photopériode de $16 \mathrm{~h}$.

Les embryons se différencient. Ils sont alors transférés par 20 en boîte de Petri, en vue de leur évolu- tion en jeune plante, sur $25 \mathrm{ml}$ d'un milieu gélosé $\mathrm{Mu}$ rashige et Skoog (1962) contenant $0,25 \mathrm{mg} . \mathrm{I}^{-1} \mathrm{de}$ $\mathrm{BAP}$ et dont les macro-éléments sont dilués de moitié (MS1/2). Les plantes obtenues sont alors repiquées en tube sur milieu MS1/2 avant d'être acclimatées en serre.

Dans nos expériences, le matériel est mis en culture dans des milieux liquides dits "chlorosants": milieu de base Nitsch et Nitsch (1969) auquel sont ajoutés $50 \mathrm{mg} . \mathrm{I}^{-1}$ de sulfate ferrique et différentes concentrations de carbonate acide de potassium (50$\left.2000 \mathrm{mg} \cdot \mathrm{l}^{-1}\right)$. L'introduction de conditions chlorosantes sera effectuée à 2 niveaux distincts du processus d'embryogenèse somatique : d'une part au niveau de la mise en culture des anthères, d'autre part au niveau des cals embryogènes.

Les coupes histologiques des cals embryogènes et des racines sont obtenues au microtome après fixation au Navashine* pendant $48 \mathrm{~h}$ puis déshydratées par des bains successifs d'alcool croissant de $70^{\circ}$ à $100^{\circ}$ avant l'inclusion dans la paraffine. On procède ensuite à un déparaffinage par des bains de toluène et d'alcool puis à une coloration de type PAS complétée par de l'hématoxyline de Groat, pour les cals embryogènes et de type Feulgen complétée par du bleu alcyan pendant 2-3 min pour les racines.

L'analyse statistique des résultats regroupés dans le tableau II est réalisée par un test $\chi^{2}$ et pour chaque ligne, les valeurs suivies d'une lettre différente sont significativement différentes au seuil de $5 \%$.

\section{RÉSULTATS ET DISCUSSION}

\section{Effets des ions bicarbonate sur la formation des embryons somatiques}

\section{Application de ces ions au stade de culture d'anthères}

Dans ces expérimentations, (tableau I) les anthères des 4 porte-greffes mises en agitation

Tableau I. Effets de la concentration en ions bicarbonate ajoutés aux milieux de cultures des anthères.

\begin{tabular}{|c|c|c|c|c|c|c|c|c|}
\hline \multirow{2}{*}{$\begin{array}{c}\text { Milieux } \\
\mathrm{KHCO}_{3}\left(m g . t^{-1}\right)\end{array}$} & \multirow{2}{*}{$\begin{array}{c}\text { Témoin } \\
0\end{array}$} & \multicolumn{7}{|c|}{ Chlorosants } \\
\hline & & 50 & 100 & 500 & 700 & 1000 & 1500 & 2000 \\
\hline & $\begin{array}{c}\text { Cals } \\
\text { embryogènes } \\
\text { Embryons }\end{array}$ & \multicolumn{3}{|c|}{$\begin{array}{l}\text { Formation de : } \\
\text { cals (3309 C) } \\
(101.14) \\
\text {. cals embryogènes } \\
\text { et embryons } \\
\text { (Rup du lot) } \\
\text { (41B) }\end{array}$} & \multicolumn{4}{|c|}{ Nécrose des anthères } \\
\hline
\end{tabular}

*Composition du Navashine: mélange en quantités égales des solutions A et B. Solution A : acide chromique $1 \mathrm{~g}$; acide acétique 10 $\mathrm{ml}$; eau distillée $85 \mathrm{ml}$. Solution B : formol $37 \% 30 \mathrm{ml}$; alcool $95^{\circ} 10 \mathrm{ml}$; eau distillée $55 \mathrm{ml}$. 
dans un milieu de Nitsch et Nitsch (1969) produisent des embryons somatiques qui proviennent d'une division des cellules diploïdes du connectif des anthères. En revanche, dans les milieux de culture contenant des concentrations élevées en ions $\mathrm{KHCO}_{3}$ (> 500 mg.l-1 c'est-à-dire 5 mmol..$^{-1}$ ) un brunissement rapide du connectif, suivi de la nécrose des anthères est observé. Pour des concentrations plus faibles, les portegreffes Rpestris du Lot et 41 B produisent des cals embryogènes (fig 1). Cependant, pour les génotypes $3309 \mathrm{C}$ et 101.14 , seule la callogénèse est observée et les coupes histologiques, réalisées au sein de ces cals, ne présentent aucune structure embryogène.

Les concentrations en ions bicarbonate supérieures à $500 \mathrm{mg.} .^{-1}$ empêchent l'obtention d'embryons somatiques quel que soit le porte-greffe étudié. Ce résultat est comparable à celui obtenu par Coulombe et al (1984a) chez le soja. D'une manière générale, l'effet des ions bicarbonate sur la chlorose des cultivars de soja est très prononcé pour des concentrations comprises entre 10 et $15 \mathrm{mmol} . .^{-1}$ (Coulombe et al, 1984b).

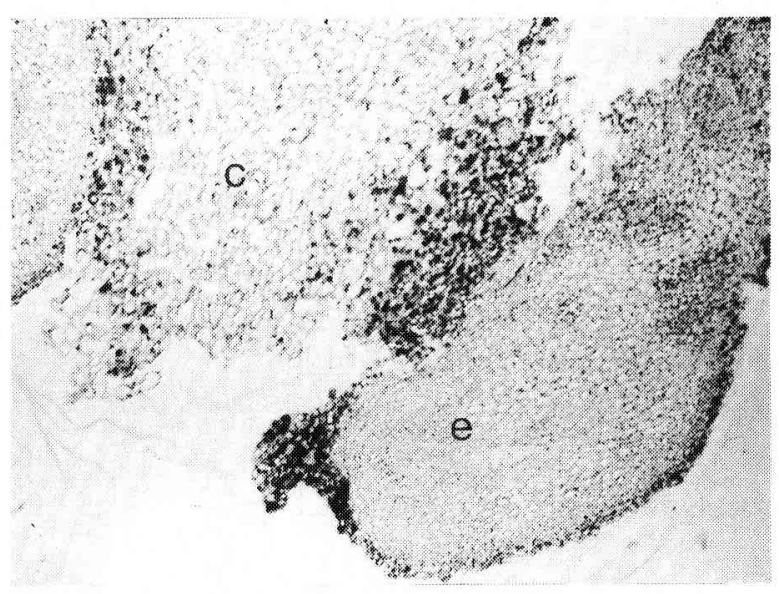

Fig 1. Coupe histologique d'un cal embryogène du portegreffe Rupestris du Lot (Grossissement: x 50). c - cal; e embryon.
Les résultats obtenus permettent d'observer que, $3309 \mathrm{C}$ et 101.14 sont les 2 porte-greffes les plus sensibles à la présence d'ions bicarbonate dans le milieu de culture des anthères. Les résultats obtenus par Chiadmi (1986) sur un test similaire portant sur des microboutures, révèlent aussi une sensibilité aux ions bicarbonate d'autant plus importante que la concentration est élevée. Le classement établi par cet auteur est comparable au nôtre : $140 \mathrm{Ru}<$ Rupestris du Lot $<3309 \mathrm{C}$. De même, ces résultats sont similaires à ceux obtenus dans le vignole par Juste et Pouget (1972) et Galet (1976). Dans les conditions expérimentales et avec les portegreffes utilisés, une bonne adéquation existe donc entre la sensibilité in vitro et celle au champ.

En revanche, si ces mêmes auteurs ont réussi à classer $41 \mathrm{~B}$ moins sensible au champ que Rupestris du Lot et 101.14 plus sensible que $3309 \mathrm{C}$, dans nos expériences il ne nous a pas été possible, pour le moment, de différencier le comportement des porte-greffes dans chaque catégorie de sensibilité. Le premier résultat est donc que Rupestris du Lot et $41 \mathrm{~B}$ sont moins sensibles à la chlorose calcaire que $3309 \mathrm{C}$ et 101.14. Cependant, l'intérêt de cette étude est de pouvoir classer les porte-greffes en 3 catégories : sensibles, résistants et intermédiaires et non pas de réaliser un classement strict puisque, même au champ, ces classements sont variables en fonction des conditions locales.

\section{Application de ces ions au cours de la culture de cals embryogènes}

Les résultats (tableau II) mettent en évidence que les très fortes concentrations en ions $\mathrm{KHCO}_{3}$ (> $1500 \mathrm{mg} . \mathrm{l}^{-1}$ ) entraînent la nécrose de tous les cals embryogènes des 4 portegreffes étudiés. En revanche, les concentrations

Tableau II. Pourcentage de germination des embryons somatiques en fonction de la concentration en ions bicarbonate.

\begin{tabular}{|c|c|c|c|c|c|}
\hline \multirow{2}{*}{$\begin{array}{l}\text { Milieux } \\
{\left[\mathrm{KHCO}_{3}\right]((\mathrm{mg} / \mathrm{l})} \\
\text { génotypes }\end{array}$} & \multirow{2}{*}{$\begin{array}{c}\text { Témoin } \\
0\end{array}$} & \multicolumn{4}{|c|}{ Chlorosants } \\
\hline & & 500 & 700 & 1000 & 1500 \\
\hline 101.14 & 5 & $\varepsilon$ & $\varepsilon$ & $\varepsilon$ & $\varepsilon$ \\
\hline $3309 \mathrm{C}$ & $15,4 \mathrm{a}$ & $7,3 \mathrm{~b}$ & $4,1 \mathrm{c}$ & $4,1 \mathrm{c}$ & $3,2 \mathrm{c}$ \\
\hline Rup du Lot & 53,8 a & $14,9 \mathrm{~b}$ & $13,6 b$ & $10,1 b$ & $3,4 \mathrm{c}$ \\
\hline $41 B$ & $14,6 \mathrm{a}$ & $12,1 \mathrm{a}$ & $10,9 \mathrm{a}$ & $6,5 \mathrm{~b}$ & $3,0 \mathrm{c}$ \\
\hline
\end{tabular}


en ions $\mathrm{KHCO}_{3} \leq 1500$ mg. $\mathrm{I}^{-1}$ permettent, pour tous les porte-greffes, non seulement la survie de tous les cals embryogènes, mais autorisent la formation d'embryons somatiques qui vont se développer et se différencier en une structure présentant un pôle apical avec ses 2 cotylédons et un rôle racinaire.

\section{Effets des ions bicarbonate sur la survie et l'évolution en jeune plante des embryons somatiques obtenus en milieux chlorosants}

Les embryons somatiques obtenus sur les milieux contenant des ions bicarbonate sont repiqués sur milieu gélosé de Murashige et Skoog (1962) pour tester leur aptitude à évoluer en jeune plante (fig 2).

Les pourcentages d'évolution en plantule des embryons somatiques obtenus pour les différents porte-greffes et pour chaque concentration en $\mathrm{KHCO}_{3}$ sont regroupés dans le tableau II.

Le très faible taux d'évolution en plantule observé pour le génotype 101.14, aussi bien sur milieu témoin que sur milieu chlorosant, ne permet pas d'en tenir compte dans l'interprétation des résultats.

Pour le génotype $41 \mathrm{~B}$, par exemple, ce taux chute à $3 \%$ lorsque la concentration en ions bi-

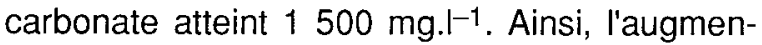

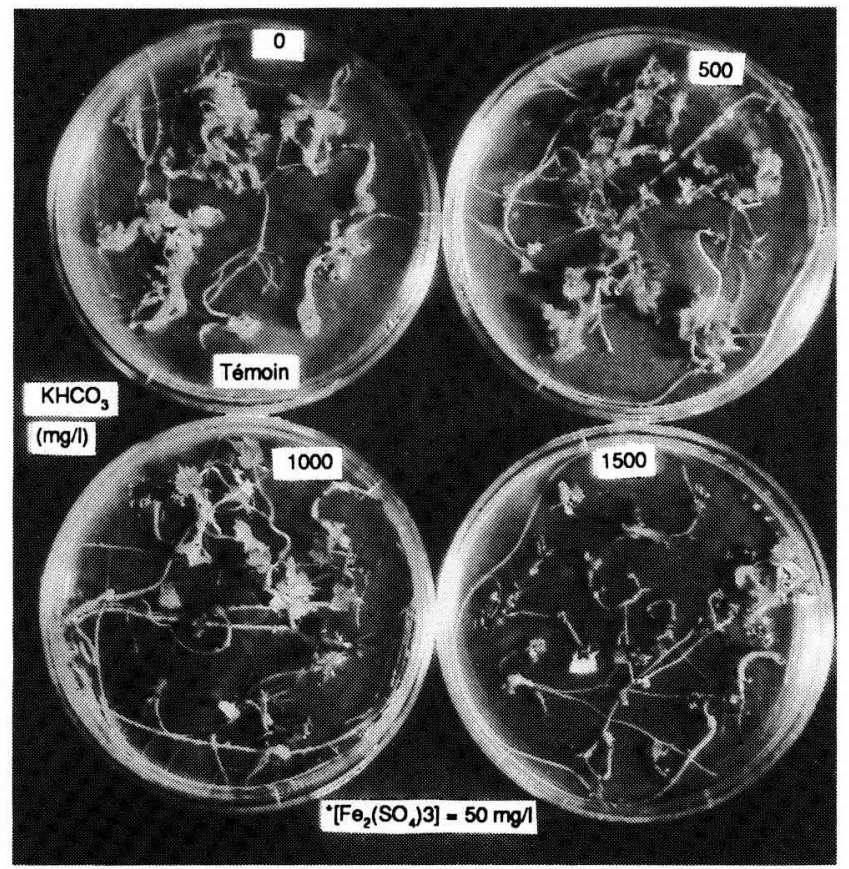

Fig 2. Évolution en plantule des embryons somatiques du porte-greffes Rupestris du Lot en présence de différentes concentrations en ions bicarbonate (grossissement $\times 0,5$ ). tation de la concentration en ions $\mathrm{KHCO}_{3}$ entraîne une baisse du taux d'évolution en plantule. De plus, en valeur absolue, pour toutes les concentrations en ions $\mathrm{KHCO}_{3}$ comprises entre 500 et $1000 \mathrm{mg}^{-1} \mathrm{l}^{-1}$, le porte-greffe $3309 \mathrm{C}$ présente le plus faible taux d'évolution en plantule à cause de nécroses des embryons somatiques, alors que les taux les plus élevés proviennent du porte-greffes Rupestris du Lot.

Cependant, par rapport au témoin de chaque génotype, nos résultats permettent de montrer que, dans les conditions expérimentales utilisées, $41 \mathrm{~B}$ ne présente une chute du pourcentage d'évolution en plantule qu'à partir d'une concentration en ions $\mathrm{KHCO}_{3}$ de $1000 \mathrm{mg} . \mathrm{l}^{-1}$, tandis que Rupestris du Lot et $3309 \mathrm{C}$ sont sensibles dès $500 \mathrm{mg} . \mathrm{l}^{-1}$. Par conséquent, le classement de la sensibilité à la chlorose calcaire in vitro est :

$$
41 \mathrm{~B}<3309 \mathrm{C}<\text { Rupestris du Lot }
$$

Ces résultats diffèrent partiellement de ceux obtenus au vignoble en 1972 par Juste et Pouget, puis en 1976 par Galet. Pour ces auteurs, Rupestris du Lot supporte plus facilement un terrain calcaire que $3309 \mathrm{C}$. Leur classement de la sensibilité à la chlorose calcaire est identique à celui réalisé par Chiadmi (1986) soit :

41 B $<$ Rupestris du Lot $<3309$ C

\section{Adaptation du matériel végétal à un milieu contenant des ions bicarbonate}

\section{Analyse du pH du milieu}

La présence d'ions bicarbonate dans les milieux de culture entraîne une élévation de $\mathrm{pH}$ dont les valeurs moyennes réalisées sur 10 mesures sont reportées dans le tableau III.

Pour des concentrations supérieures à 1000 mg. $\mathrm{I}^{-1}$, les valeurs du $\mathrm{pH}$ ne sont pas mentionnées dans le tableau, puisque on observe une limitation de l'embryogenèse dès $1000 \mathrm{mg} \cdot \mathrm{l}^{-1}$.

Tableau III. Valeurs du pH des milieux de culture en fonction de la concentration en ions bicarbonate.

\begin{tabular}{lllll}
\hline$\left[\mathrm{KHCO}_{3}\right]\left(\mathrm{mg.} \mathrm{I}^{-1}\right)$ & 0 & 500 & 700 & 1000 \\
\hline Valeurs du pH & 5,35 & 7,72 & 7,83 & 8,02
\end{tabular}


Les valeurs mesurées, d'une moyenne de 7,72 lorsque la concentration en $\mathrm{KHCO}_{3}$ est de 500 mg..$^{-1}$ atteignent 8,02 pour une concentration de $1000 \mathrm{mg} . \mathrm{I}^{-1}$. En culture in vitro, le $\mathrm{pH}$ des milieux utilisés est en général voisin de 5,7.

Il est alors intéressant de s'interroger sur la stratégie d'adaptation de notre matériel végétal à de telles conditions de $\mathrm{pH}$. À cet effet, l'évolution de la valeur du $\mathrm{pH}$ en fonction du temps et de la concentration en ions bicarbonate est présentée pour le porte-greffe Rupestris du Lot (fig 3).

En présence d'ions bicarbonate, une baisse importante du $\mathrm{pH}$ est observée dès le 1er j de la culture, et se poursuit jusqu'à atteindre la valeur voisine de 5,2 observée chez le témoin le $6^{e}$ jour. Ces résultats sont similaires pour $3309 \mathrm{C}$ où, à la concentration en ions bicarbonate de 1000 mg. $\left.\right|^{-1}$, la valeur du $\mathrm{pH}$ évolue de 8,02 à 5,64 au curs des 6 premiers jours de culture. Ces chutes de $\mathrm{pH}$ très rapides peuvent s'expliquer par une extrusion de protons qui acidifieraient ainsi le milieu jusqu'à un équilibre nécessaire à la survie des cellules végétales. Ces protons seraient d'autant plus excrétés dans le milieu de culture que la valeur initiale du $\mathrm{pH}$ serait élevée, c'est-àdire que la concentration en ions bicarbonate est importante. Cependant à partir de la concentration très élevée de $1000 \mathrm{mg} . \mathrm{I}^{-1}$, le milieu subit une acidification moins importante. L'inhibition de l'extrusion des protons pourrait résulter d'un début de toxicité dûe aux concentrations élevées en ions bicarbonate vis-à-vis du matériel végétal. Römheld (1987) pense que chez les plantes croissant en sol calcaire et présentant une concentration élevée en ions bicarbonate, la capacité de la pompe à protons pourrait être insuffisante pour générer un gradient de $\mathrm{pH}$ suffisant

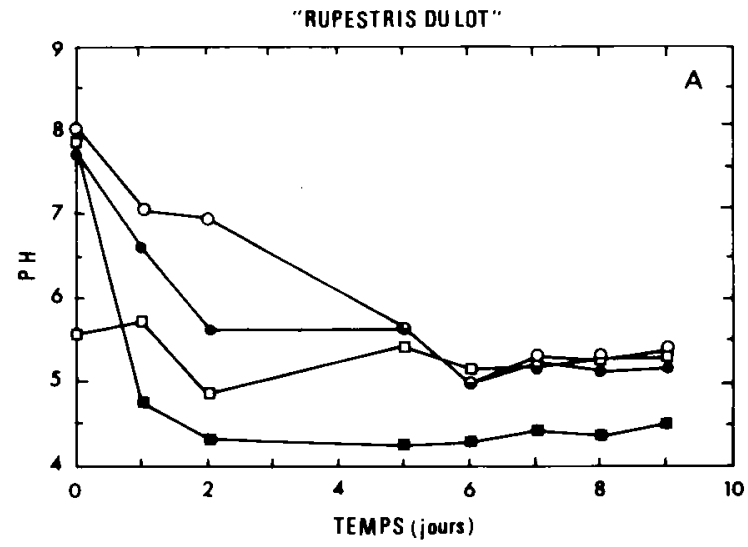

Fig 3. Évolution des valeurs du $\mathrm{pH}$ en fonction du temps en présence de concentrations en ions bicarbonate variant de 0 à $1000 \mathrm{mg} . \mathrm{H}^{-1} . \square-$ Témoin - concentrations en ions $\mathrm{KHCO}_{3}=$ $0 \mathrm{mg} . \mathrm{I}^{-1} ; 0-$ concentration en ions $\mathrm{KHCO}_{3}=500 \mathrm{mg} \cdot \mathrm{I}^{-1} ; \mathbf{D}-$ cncentration en ions $\mathrm{KHCO}_{3}=700 \mathrm{mg} . \mathrm{I}^{-1} ; \mathrm{O}-$ concentration en ions $\mathrm{KHCO}_{3}=1000 \mathrm{mg} . \mathrm{I}^{-1}$. pour stimuler le système impliqué dans la réduction du fer.

\section{Étude de la structure de la racine}

Chez la vigne, l'évolution des embryons somatiques et l'obtention de jeunes plantules adaptées aux milieux basiques chlorosants (fig 4) par acidification du milieu, entraîne-t-elle une modification structurale des racines?

Pour répondre à cette question, des coupes histologiques de racines ont été effectuées et comparées à celles des plantes témoins (fig $5 \mathrm{~A}$, B).

L'observation de ces coupes tend à montrer qu'il existe une adaptation particulière des racines liée aux conditions de culture chlorosantes. Elle se traduit par un épaississement de l'épiderme cortical racinaire. Ce renforcement provient essentiellement d'un épaississement des parois cellulaires, plutôt que d'une augmentation du nombre des cellules. En effet, l'épiderme est constitué d'une seule couche de cellules, comme chez les plantes témoins.

Chez la vigne, pour les porte-greffes étudiés, et dans nos conditions de culture in vitro, l'aptitude des embryons somatiques à se développer en milieux chlorosants s'exprime morphologiquement au niveau racinaire. Ces résultats corroborent ceux observés par Bavaresco (1989) sur d'autres porte-greffes de vigne cultivés en terrains calcaires. Cet auteur observe alors un système racinaire beaucoup moins développé que

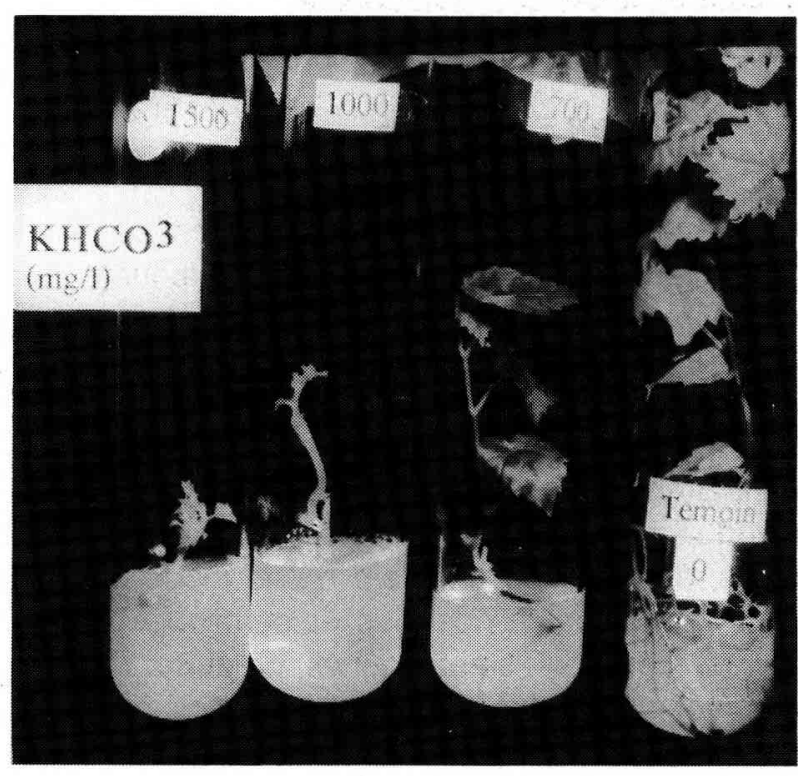

Fig 4. Croissance de jeunes plantules de Rupestris du Lot en présence de différentes concentrations en ions bicarbonate. 


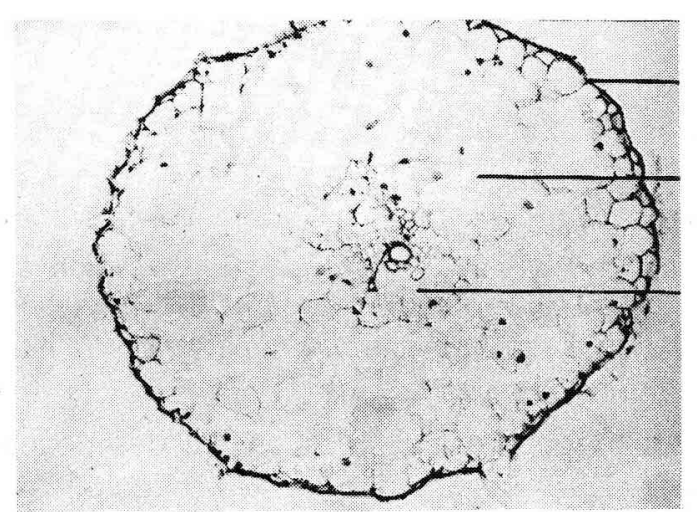

A

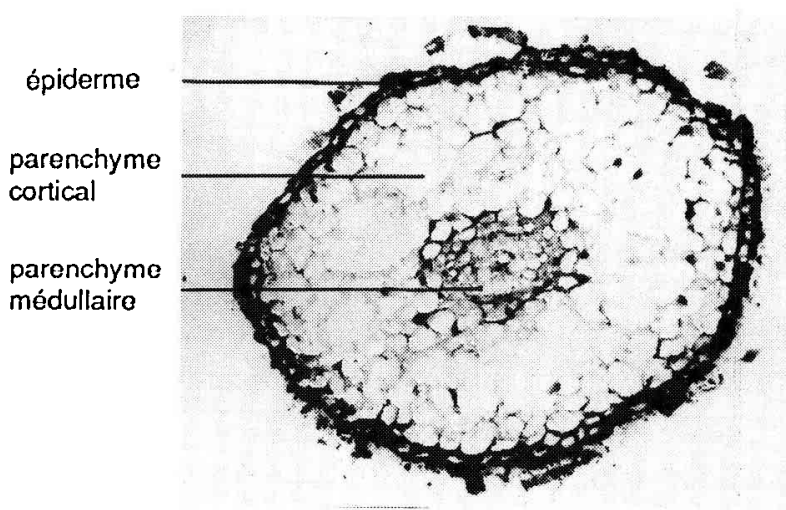

B

Fig 5. Coupes transversales dans la zone apicale racinaire du porte-greffes Rupestris du Lot. A - milieu témoin, concentration en ions $\mathrm{KHCO}_{3}=0 \mathrm{mg} \cdot \mathrm{l}^{-1} ; \mathrm{B}$ - milieu chlorosant, concentration en ions $\mathrm{KHCO}_{3}=1000 \mathrm{mg} . \mathrm{I}^{-1}$.

celui des plantes cultivées sur des sols non chlorotiques, cependant cette observation morphologique n'est pas étayée par des coupes histologiques. Nos résultats se rapprochent également de ceux observés par de nombreux auteurs : Kramer et al (1980); Bienfait et al (1987) sur le tournesol tout comme Römheld (1987) sur la pomme de terre, qui constatent une modification de la structure des cellules racinaires. II se formerait, dans la zone apicale des racines, des cellules de transfert épidermiques dont la présence serait corrélée à une sortie de protons. $\mathrm{Ce}$ phénomène, observé jusqu'à présent sur des plantes herbacées annuelles (tournesol, soja, carotte, pomme de terre) semble donc exister également chez les végétaux ligneux (vigne) en tant que stratégie d'adaptation à un milieu de culture riche en ions bicarbonate.

\section{CONCLUSION}

Les résultats expérimentaux obtenus ont permis de montrer que l'addition d'ions bicarbonate à des faibles concentrations dans le milieu de culture n'inhibe pas l'embryogenèse somatique. Les cultivars $3309 \mathrm{C}$ et 101.14 se sont révélés sensibles à la chlorose calcaire en culture in vitro, contrairement à 41 B et Rupestris du Lot. L'adaptation de ces clones à des milieux chlorosants, se fait simultanément à une modification de la structure des cellules épidermiques racinaires. Les cals embryogènes produisent des embryons capables de germer sur des milieux contenant de très fortes concentrations en ions bicarbonate (1 $000{\mathrm{mg} . \mathrm{I}^{-1}}^{-1}$. De plus, sur la base des 4 porte-greffes étudiés, un parallélisme est noté entre le classement établi au vignoble et celui observé in vitro. Cependant, ces premiers résultats ne permettent pas de distinguer s'il s'agit d'une adaptation physiologique ou d'une résistance obtenue par variation somaclonale de nature héréditaire. Le passage en sol calcaire des clones sélectionnés présentant une certaine tolérance in vitro est en cours de réalisation.

\section{REMERCIEMENTS}

Ce travail a été financé par le ministère de la Recherche et de l'Enseignement supérieur dans le cadre d'un contrat de recherche avec les établissements Moët et Chandon (Programme national vigne). Les auteurs remercient Mme $O$ Robin pour la réalisation des coupes histologiques.

\section{RÉFÉRENCES}

Bavaresco L (1989) Investigations about some physiological parameters involved in chlorosis occurence in different grapevine rootstocks and in a $V$ vinifera variety. $V$ int Symp grape breeding. Allemagne

Bienfait HF, De Weger LA, Kramer D (1987) Control of the development of iron-efficiency reactions in potato as a response to iron deficiency is located in the roots. Plant Physiol 83, 244-247

Boxma R (1972) Bicarbonates as the most important soil factor in lime-induced chlorosis in the Netherlands. Plant Soil 37, 233-243

Brown JC, Chaney RL, Ambler JE (1971) A new tomato mutant inefficient in the transport of iron. Physiol Plant 25, 48-53 
Chiadmi N (1986) Culture in vitro de la vigne (Vitis $\mathrm{sp}$ ). Régénération de plantes à partir d'un fragment de limbe foliaire. Mise au point d'un test précoce de résistance à la chlorose calcaire. Thèse Doc $3^{e}$ cycle Univ Paris XI, Orsay, $123 p$

Coulombe BA, Chaney RL, Wiebeld WJ (1984a) Bicarbonate directly induces iron chlorosis in susceptible soybean cultivars. Soil Sci Soc Am J 48, 1297-1301

Coulombe BA, Chaney RL, Wiebold WJ (1984b) Use of bicarbonate in screening soybeans for resistance to iron chlorosis. J Plant Nutr 7, 411-425

Fairbanks DJ, Orf JH, Inskeep WP, Bloom PR (1987) Evaluation of soybean genotypes for iron deficiency chlorosis in potted calcareous soil. Crop Sci 27, 953-957

Galet P (1976) Précis d'ampélographie pratique 4e éd, Montpellier, $226 \mathrm{p}$

Juste C, Pouget R (1972) Appréciation du pouvoir chlorosant des sols par un nouvel indice faisant intervenir le calcaire actif et le fer facilement extractible. Application au choix des porte-greffes de la vigne. CR Séances Agric Fr 58, 352-357

Kramer D, Romheld V, Landsberg E, Marschner $H$ (1980) Induction of transfer-cell formation iron deficiency in the root epidermis of Helianthus annuus $L$. Springer-Berlag, Berlin
Mayer $G$ (1985) Influence of soluble iron and concentration of $\mathrm{HCO}_{3}$ in nutritive solution on growth and occurence of chlorosis in rooted cuttings of different vine cultivars. $4^{e}$ Symp Int Gen Vigne Verone

Mullins MG (1966) Test-plants for investigations of the physiology of fruiting in Vitis vinifera L. Nature 209, 419-420

Murashige T, Skoog F (1962) A revised method for rapid growth and bioassays with tobacco tissue cultures. Physiol Plant 15, 473-497

Murisier F, Briguet C (1988) Rendement et chlorose de la vigne. Rev Suisse Vitic Arboric Hortic 20, 165-172

Nitsch JP, Nitsch C (1969) Haploid plants from pollen grains. Science $163,85-87$

Pouget R, Ottenwaelter M (1973) Étude méthodologique de la résistance à la chlorose calcaire chez la vigne : principe de la méthode des greffages réciproques et application à la recherche de portegreffes résistants. Ann Amélior Plant 23, 347-356

Römheld V (1987) Different strategies for iron acquisition in higher plants. Physiol Plant 70, 231-234

Saglio P (1969) Nutrition en fer de la vigne. I. Essai d'induction d'une chlorose ferrique par l'action combinée du bicarbonate et de l'orthophosphate sur 2 variétés : l'une sensible et l'autre résistante. Ann Physiol Veg 11, 27-35 